\title{
The impact of emergency medical services in acute heart failure
}

\author{
Harjola, Pia
}

2017-04-01

Harjola , P , Boyd , J , Tarvasmäki , T , Mattila , J , Koski , R, Kuisma , M \& Harjola , V-P 2017 , ' The impact of emergency medical services in acute heart failure ' , International Journal of Cardiology , vol. 232 , pp. 222-226 . https://doi.org/10.1016/j.ijcard.2017.01.017

http://hdl.handle.net/10138/237085

https://doi.org/10.1016/j.ijcard.2017.01.017

publishedVersion

Downloaded from Helda, University of Helsinki institutional repository.

This is an electronic reprint of the original article.

This reprint may differ from the original in pagination and typographic detail.

Please cite the original version. 


\title{
The impact of emergency medical services in acute heart failure
}

\author{
Pia Harjola, James Boyd, Tuukka Tarvasmäki, Juho Mattila, Reijo Koski, Markku Kuisma, Veli-Pekka Harjola * \\ Emergency Medicine, University of Helsinki, Department of Emergency Medicine and Services, Helsinki University Hospital, Helsinki, Finland, Haartmaninkatu 4, FI-00290 Helsinki, Finland
}

\section{A R T I C L E I N F O}

\section{Article history:}

Received 31 August 2016

Received in revised form 30 December 2016

Accepted 3 January 2017

Available online 4 January 2017

\section{Keywords:}

Acute heart failure

Emergency medical services

Prehospital management

Emergency department

\begin{abstract}
A B S T R A C T
Background: Real-life data on the role of emergency medical services (EMS) in acute heart failure (AHF) are scarce. Our aim was to describe prehospital treatment of AHF and to compare patients using EMS with selfpresented, non-EMS patients.

Methods: Data were collected retrospectively from three university hospitals in Helsinki metropolitan area between July 1, 2012 and July 31, 2013. According to the use of EMS, patients were divided into EMS and nonEMS groups.

Results: The study included 873 AHF patients. One hundred were (11.5\%) EMS and 773 (88.5\%) non-EMS. EMS patients more often had comorbidities. Initial heart rate $(\mathrm{HR})$ and peripheral oxygen saturation $\left(\mathrm{SpO}_{2}\right)$ differed between EMS and non-EMS patients; mean HR 89.2 (SD 22.5) vs. $83.7(21.5) / \mathrm{min}(\mathrm{p}=0.02)$ and $\mathrm{SpO}_{2} 90.3$ (8.6) vs. $92.9(6.6) \%(p=0.01)$. However, on presentation to ED EMS patients' vital signs were similar to nonEMS patients'. On presentation to ED $46.0 \%$ were normotensive and $68.2 \%$ "warm and wet". Thirty-four percentage of EMS patients received prehospital medication. In-hospital mortality was $6.0 \%$ and $7.1 \%(\mathrm{p}=0.84)$ and length of stay (LOS) 7.7 (7.0) and 8.5 (7.9) days $(\mathrm{p}=0.36$ ) in EMS and non-EMS groups.

Conclusion: The use of EMS and administration of prehospital medication was low. EMS patients had initially worse $\mathrm{HR}$ and $\mathrm{SpO}_{2}$ than non-EMS patients. However, EMS patients' signs improved and were similar on presentation to ED. There were no differences in in-hospital mortality and LOS. This underscores the need for equal attention to any AHF patient independent of the arrival mode.
\end{abstract}

(c) 2017 Elsevier Ireland Ltd. All rights reserved.

\section{Introduction}

Acute heart failure (AHF) is one of the most common reasons for hospital admission among elderly people. However, only a minority of these patients uses emergency medical services (EMS) [1].

The role of early treatment of AHF, including prehospital care, has been emphasized noticeably in the recently published 2016 ESC Guidelines for the diagnosis and treatment of acute and chronic heart failure (HF) as well as in the recommendations on prehospital and early hospital management of AHF $[2,3]$. However, there is a significant lack of knowledge on the actual characteristics of prehospital management of AHF and on the differences between patients arriving by ambulance and those self-presenting into emergency department (ED). The literature on prehospital AHF treatment mainly focuses on the treatment of severe AHF conditions such as non-invasive ventilation (NIV) in acute pulmonary oedema, the second most common manifestation of AHF

\footnotetext{
* Corresponding author at: Emergency Medicine, University of Helsinki, Division of Emergency Medicine, Department of Emergency Medicine and Services, Helsinki University Hospital, Helsinki, Finland.

E-mail address: veli-pekka.harjola@hus.fi (V.-P. Harjola).
}

[4-6]. The data on the effectiveness and safety of prehospital medications is scarce.

The aim of this study was first to describe the implementation and effects of EMS in AHF patients. Second we wanted to compare the clinical characteristics of patients arriving to the ED by ambulance to those self-presenting as well as their length of hospital stay (LOS) and inhospital mortality.

\section{Material and methods}

In this retrospective multicentre study, we included all patients admitted to the ED with a primary discharge diagnosis of congestive HF. Data were collected from three university hospitals in the Helsinki metropolitan area between July 1, 2012 and July 31, 2013. Patients transferred to the ED from another hospital were excluded. According to the use of EMS, patients were divided into EMS and self-presenting, non-EMS groups.

Prehospital and hospital data were collected from the regional clinical information system. The prehospital data were collected from the Merlot Medi electronic patient reporting system (CGI, Finland) and the in-hospital data from the Uranus electronic patient information system (CGI, Finland). The data included patient history, prehospital and in-hospital management, the first prehospital vital signs (heart rate (HR), systolic blood pressure (SBP), peripheral oxygen saturation $\left(\mathrm{SpO}_{2}\right)$, and respiratory rate $(\mathrm{RR})$ ), vital signs on presentation to ED, and LOS and in-hospital mortality. These variables were compared between the non-EMS and EMS group. 
AHF was diagnosed according to the following criteria: clinical symptoms and signs of systemic or pulmonary venous congestion and/or hypoperfusion. In addition, chest X-ray was evaluated for congestion, interstitial or alveolar pulmonary oedema, and pleural effusion. According to these findings patients were classified into four different clinical profiles (warm and wet, warm and dry, cold and wet, and cold and dry) [7]. Results from the most recently done echocardiogram were also evaluated. The patients were divided into three groups according to their left ventricular ejection fraction (LVEF) $(\mathrm{EF}<40 \%$, reduced $\mathrm{EF}$; EF $40-49 \%$, mid-range $\mathrm{EF}$ and $\mathrm{EF} \geq 50 \%$, preserved $\mathrm{EF}$ ).

All patients were categorized into four groups according to their SBP on presentation to ED: Group 1 (SBP $<100 \mathrm{~mm} \mathrm{Hg}$ ), group 2 (SBP $100-119 \mathrm{~mm} \mathrm{Hg}$ ), group 3 (SBP 120-160 mm Hg), and group 4 (SBP > $160 \mathrm{~mm} \mathrm{Hg}$ )

The emergency-dispatching centre assessed the urgency and complaints of EMS patients. According to local EMS guidelines the urgency is categorized as A, B, C, or D according to the recommended response time from call to EMS arrival on site. In categories A and B the upper limit of the response time is $8 \mathrm{~min}$, in category C $30 \mathrm{~min}$ and in category D $120 \mathrm{~min}$. The level of EMS unit also differs in these four categories. In category A, the patient is taken care by the highest advanced life support (ALS) unit available. ALS units contain continuous positive airway pressure (CPAP) equipment, intravenous morphine, nitrate infusion and intravenous beta-blocker (metoprolol) among other advanced treatments. In categories B, C, and D, the basic life support (BLS) unit first assesses the patient and evaluates the possible need for a more advanced unit and/or physician on site. BLS units are equipped with supplementary oxygen, crystalloid infusion, nitrate spray, and acetylsalicylic acid, as well as defibrillator and intubation equipment. The BLS units do not contain any intravenous medication or CPAP equipment. If needed a physician staffed mobile intensive care unit (MICU) is sent on site. Due to the small number of patients in category $A$ and the equal response time with category $B$, these two categories were combined for statistical analyses.

For study purposes, the main complaints recorded by the dispatching centre were categorized into five major groups: a) dyspnoea, b) general weakness, c) chest pain d) transportation from nursing home or primary health care, and e) other complaints.

Four time points (call to the dispatching centre, EMS arrival on site, departure from the site, and patient presentation to the ED) were collected from the EMS database, and corresponding time intervals were analysed.

Mortality difference between EMS and non-EMS patients was further analysed with propensity score matching [8]. Nearest neighbour 1:1 matching with a caliper width 0.2 of the standard deviation (SD) of the propensity score without replacement was used. Propensity score was estimated with included potential confounders of mortality: age, gender, medical history (chronic heart failure (CHF), coronary artery disease, previous history of acute myocardial infarction, previous history of coronary artery bypass graft surgery, hypertension, diabetes, cerebrovascular disease, chronic kidney disease, and chronic obstructive pulmonary disease), and initial presentation (SBP, $\mathrm{HR}$, and $\mathrm{SpO}_{2}$ ). Initial RR (data missing in $25 \%$ ) was included in the propensity score estimation in a separate sensitivity analysis with all the variables above. Balance between matched groups was assessed with standardized mean differences (SMD).

The categorical variables are presented in numbers ( $\mathrm{n}$ ) and percentages (\%), and continuous variables in means with the SD. Time is presented as the mean and SD in hours and minutes (hh:mm). The threshold p-value for statistical significance was 0.05 . Statistical analyses were performed using SPSS 22.0 statistical software (IBM Corp., Armonk, NY, USA). The study had the institutional approval of Helsinki University Hospital.

\section{Results}

Our study included 873 patients: 100 (11.5\%) in the EMS group and $773(88.5 \%)$ in the non-EMS group. The patient characteristics are presented in Table 1. The mean age of all patients was 75.7 (11.7) years $[9,10]$. The proportions of females and those with a history of diabetes were slightly but not significantly higher in the EMS group compared to the non-EMS group. However, a significantly greater proportion of EMS patients had a previous history of $\mathrm{CHF}$ ( $\mathrm{p}=0.048$ ), acute myocardial infarction $(p=0.042)$, coronary artery disease $(p=0.012)$, and chronic kidney disease $(\mathrm{p}<0.001)$. The mean LVEF of all patients was $46.3 \%$ (SD 16.0). In total $26.3 \%$ of patient had reduced LVEF and $34.2 \%$ of patients had preserved LVEF. The majority of patients had midrange LVEF. The LVEF did not differ between EMS and non-EMS patients $(\mathrm{p}=0.525)$ (Table 1.).

The major complaints were dyspnoea $(\mathrm{n}=38,38.0 \%)$ and general weakness ( $n=27,27.0 \%)$, followed by chest pain ( $n=9,9.0 \%)$. Twenty-six (26.0\%) patients had other complaints or were transportations to the ED from a nursing home or primary health care unit. Regarding urgency categories, three (3.0\%) patients were in urgency category A, 30 (30.0\%) in category B, 46 (46.0\%) in category C, and 20 (20.0\%) in category D. BLS units took care of $57 \%$ of all EMS patients, and ALS units took care of $39 \%$. A MICU took care of $4 \%$ of all patients.

Eighty-four percentages of all dispatched EMS units were on site within the recommended response time. The mean response time was
0:19 (0:18) (Table 2). The recommended response times were achieved in $97.7 \%$ of cases in category $C$, and in all cases in category D. However, in categories A and B, only $54.8 \%$ of cases reached the recommended response time.

The prehospital and ED presentation vital signs as well as SBP classes are presented in Table 3. Thirty-three percentages of EMS patients had prehospital $\mathrm{SpO}_{2}$ on room air $<90 \%$. Fifty percentages of these patients were taken care by ALS units. Clinically and statistically the main finding was a decrease in the patients' mean HR from 89.2 beats per minute (bpm) on site to $84.5 \mathrm{bpm}$ on presentation to the ED ( $\mathrm{p}=0.024$ ).

The chest x-ray findings of all patients were as follows: $61.9 \%$ had pulmonary congestion, $12.8 \%$ interstitial pulmonary oedema, $3.6 \%$ alveolar pulmonary oedema, and $47.2 \%$ had pleural effusion. Jugular venous pressure was elevated in $12.8 \%$ of patients, and peripheral oedemas were reported in $47.4 \%$. According to the clinical criteria $68.2 \%$ of AHF patients were profiled as warm and wet, $13.9 \%$ warm and dry, another $13.9 \%$ as cold and wet, and $2.5 \%$ as cold and dry. The profile of cold and dry was more common in the EMS group $(p=0.009)$. No statistical differences were observed in the three other clinical profiles.

Prehospital medication was administered to $34 \%$ of EMS patients. Fifty percentage of de novo and $28.4 \%$ of CHF patients received prehospital medication. The administered prehospital treatments included intravenous morphine, intravenous metoprolol, nitrate spray, nitrate infusion, and a combination of inhaled ipratropium bromide and salbutamol. Twenty-one (21.0\%) patients received intravenous morphine. A similar proportion received nitrate spray. Of these 21 patients, 11 suffered from chest pain and 17 were hypertensive (SBP > $140 \mathrm{~mm} \mathrm{Hg}$ ). Metoprolol was given to nine (9.0\%) patients, nitrate infusion to seven (7.0\%), and a combination of ipratropium bromide and salbutamol to another seven (7.0\%). Intravenous morphine was administered in a mean cumulative dose of 4.7 (2.2) $\mathrm{mg}$ and metoprolol 2.3 (1.3) $\mathrm{mg}$. The maximum rate of nitrate infusion was $1.1(0.2) \mathrm{mg} / \mathrm{h}$. None of the patients received prehospital CPAP treatment.

Mean LOS of the total study population was 8.4 (7.8) days, with no significant difference observed between EMS (7.7 (7.0) days) and non-EMS patients (8.5 (7.9) days) $(p=0.363)$. Likewise, the inhospital mortalities were similar, being $6.0 \%$ in the EMS group and $7.1 \%$ in the non-EMS group ( $\mathrm{p}=0.836$ ). In addition, in-hospital mortality between the two groups was assessed with propensity score matching. The matching procedure resulted in 87 matched pairs; no significant imbalances between the groups remained with respect to propensity score (SMD 0.006), the covariates used (SMD $<0.12$ for all) or the average of absolute values of SMDs of covariates (0.056). After propensity score matching, the result remained similar in the two groups: $6.9 \%$ in EMS vs. 8.0\% in non-EMS ( $p=0.8$ ). Sensitivity analysis including initial RR in the propensity score estimation showed a similar result.

\section{Discussion}

This study described the current practices in the prehospital AHF management. Secondly, it illustrated the characteristics of EMS and non-EMS patients. Our first finding was that a rather small proportion of AHF patients used ambulance. These patients more often had a history of CHF, coronary artery disease, acute myocardial infarction, and chronic kidney disease. Secondly, we discovered that few patients received medication in EMS. Third, EMS patients were initially more unstable but their vital signs improved before presentation to ED. However, the prevalence of dry and cold patients was significantly higher in the EMS group. Lastly, the LOS and in-hospital mortality were similar in EMS and non-EMS groups.

Our first finding, that only one-tenth of AHF patients used EMS is somewhat surprising. Similarly, a previous study showed that AHF patients were more likely to self-present to the ED [1]. Patients presenting with worsening CHF more often arrived by ambulance to the ED than those with de novo AHF. The proper use of EMS among de novo patients may be limited due to difficulties in recognizing the alarming symptoms 
Table 1

Patient characteristics, $\mathrm{n}(\%)$.

\begin{tabular}{|c|c|c|c|c|}
\hline & All, $\mathrm{n}=873(100.0)$ & EMS patients, $\mathrm{n}=100$ (11.5) & Non-EMS patients, $\mathrm{n}=773$ (88.5) & P-value \\
\hline Age, mean (SD) & $75.7(11.7)$ & $76.2(10.8)$ & $75.6(11.9)$ & 0.621 \\
\hline Female & $423(48.5)$ & $55(55.0)$ & $368(47.6)$ & 0.169 \\
\hline \multicolumn{5}{|l|}{ Medical history } \\
\hline Chronic heart failure & $568(65.1)$ & $74(74.0)$ & $494(63.9)$ & 0.048 \\
\hline Coronary artery disease & $335(38.4)$ & $50(50.0)$ & $285(36.9)$ & 0.012 \\
\hline Previous myocardial infarction & $138(15.8)$ & $23(23.0)$ & $115(14.9)$ & 0.042 \\
\hline CABG & $134(15.3)$ & $23(23.0)$ & $111(14.4)$ & 0.038 \\
\hline Hypertension & $556(63.8)$ & $60(68.0)$ & $488(63.2)$ & 0.378 \\
\hline Diabetes mellitus & $374(42.8)$ & $50(50.0)$ & $324(41.9)$ & 0.133 \\
\hline Chronic atrial fibrillation & $384(44.0)$ & $47(47.0)$ & $337(43.6)$ & 0.523 \\
\hline Cerebrovascular disease & $125(14.3)$ & $13(13.0)$ & $112(14.5)$ & 0.763 \\
\hline Significant valvular disease & $55(6.3)$ & $10(10.3)$ & $51(6.7)$ & 0.208 \\
\hline Chronic kidney disease & $170(19.5)$ & $38(38.0)$ & $132(17.1)$ & $<0.001$ \\
\hline History of DCMP & $90(10.3)$ & $10(10.0)$ & $80(10.3)$ & 1.000 \\
\hline COPD & $136(15.6)$ & $20(20.0)$ & $116(15.0)$ & 0.190 \\
\hline $\operatorname{LVEF}(\%)$, mean $(S D)(n=760)$ & $46.3(16.0)$ & $47.3(15.9)$ & $46.1(16.0)$ & 0.525 \\
\hline
\end{tabular}

DCMP, dilated cardiomyopathy; COPD, chronic obstructive pulmonary disease; CABG, coronary artery bypass graft; LVEF, left ventricular ejection fraction.

[11,12]. Therefore, patient education should receive appropriate attention. In addition, EMS patients more often had chronic cardiac illnesses, which most likely improve their awareness of warning signs and symptoms. Finally, patient's subjective judgment of symptom severity varies and alters the threshold for seeking medical attention.

The most frequently assessed urgency category by the emergencydispatching centre was category $\mathrm{C}$, in which the recommended response time ( $<30 \mathrm{~min}$ ) was well achieved. The average EMS response time in our study was clearly shorter compared to a previous study, in which the median response time was over three-fold [12]. An explanation for the short response time might be the relatively short distances and light traffic in the Helsinki metropolitan area. However, in only slightly more than half of the most urgent EMS calls, the response times were within $8 \mathrm{~min}$. It has been reported that only when the transportation time exceeds 45 min the risk of in-hospital mortality increases significantly [12]. In our study the transportation time was clearly shorter, and in line with previous data in the literature [12-14].

The present study reveals that in this large urban area, the administration of prehospital treatment is actually rare. Prehospital medication was administered to only one-third of EMS patients. This could be explained with the rather short transportation times and distances to the hospital in the Helsinki metropolitan area. It has been pointed out that it might be challenging for paramedics to diagnose AHF $[15,16]$. Consequently, patient's earlier diagnoses might have a major role in guiding the prehospital treatment. Still, in the present study de novo patients received pre-hospital medication more often compared to CHF patients. One explanation might be that due to the uncertain cause of the presenting symptoms paramedics were more prone to give symptom-based medication to de novo patients.

The most frequently administered medications were intravenous morphine and nitrate spray, which is not surprising as dyspnoea and chest pain were among the main complaints. Still, only one-fifth of EMS patients received these two medications. The administration of intravenous morphine has been reported to be even scarcer, only $6 \%$ receiving it $[17,18]$. In addition, guidelines recommend to use morphine in severe AHF, accompanied by anxiety and dyspnoea [2]. Patients most likely to benefit from medical therapy are those with hypertension and/or significant hypoxemia $[17,19]$. The frequent administrations of nitrate spray could be explained with the high prevalence of coronary artery disease. Nitrate infusion was administered to less than onetenth of the patients. Intravenous beta-blocker was also administered to only a few patients.

AHF patients are known to have airway constriction in their small airways, i.e. cardiac asthma. In theory that justifies the use of inhaled bronchodilators as first aid though data on this topic is conflicting [20-22]. Anyhow, in our study less than one tenth of patients received bronchodilator. On the other hand, it has been reported that supplementary oxygen, semi-recumbent positioning and monitoring of vital signs on their own could be sufficient prehospital treatment $[4,17]$. Moreover, some data have shown that the addition of pharmacological treatment to CPAP in a prehospital setting results in no measurable improvement among AHF patients compared with CPAP alone [5].

It is recommended to use CPAP when there is a clinical suspicion of pulmonary oedema. A previous study demonstrated that prehospital CPAP treatment has a positive effect on $\mathrm{RR}, \mathrm{HR}, \mathrm{SpO}_{2}$, and $\mathrm{SBP}$ in patients suffering from pulmonary oedema [6]. However, none of our patients received CPAP treatment though one third of the patients were hypoxemic. The use of CPAP was also reported to be rare in a previous study, in which only $1 \%$ of patients received the treatment [23].

Not surprisingly, EMS patients' status was initially less stable than the non-EMS patients'. No significant improvement was observed in EMS patients' vital signs, expect from HR, from site to the ED presentation. In contrast, a previous study reported an improvement in patients' vital signs ( $\mathrm{SBP}$, mean arterial pressure, $\mathrm{HR}$, RR, and $\mathrm{SpO}_{2}$ ) after prehospital treatment [17]. However, these patients were initially less stable than those in our study. This particular study suggested that the level of improvement in vital signs is dependent on the initial values. Thus, the more stable initial status along with infrequent administration of prehospital medication could explain the smaller improvement in vital signs in our study. On presentation to the ED no significant differences were observed in vital signs between EMS and non-EMS patients. However, dry and cold profile was more common in EMS compared to

Table 2

Urgency categories in emergency medical services.

\begin{tabular}{|c|c|c|c|c|}
\hline Urgency category & All, $\mathrm{n}=100$ & Categories $\mathrm{A}$ and $\mathrm{B}, \mathrm{n}=33$ & Category $C, n=46$ & Category $\mathrm{D}, \mathrm{n}=20$ \\
\hline Response time from call to site & $0: 19(0: 18)$ & 0:08 (0:03) & $0: 17(0: 10)$ & $0: 40(0: 26)$ \\
\hline Time spent on site & $0: 28(0: 16)$ & $0: 35(0: 09)$ & $0: 26(0: 18)$ & $0: 21(0: 15)$ \\
\hline Transportation time & $0: 19(0: 30)$ & $0: 20(0: 37)$ & $0: 16(0: 25)$ & $0: 25(0: 30)$ \\
\hline Total time from call to hospital presentation & $1: 14(1: 02)$ & $1: 04(0: 32)$ & $1: 14(1: 18)$ & $1: 37(1: 02)$ \\
\hline
\end{tabular}

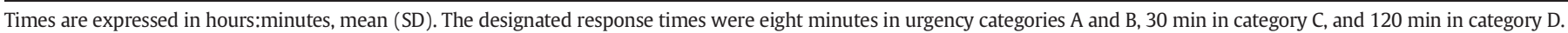


Pre-hospital and admission vital signs and blood pressure classes on admission.

\begin{tabular}{|c|c|c|c|}
\hline & $\begin{array}{l}\text { 1. First pre-hospital values of the EMS } \\
\text { group }\end{array}$ & $\begin{array}{l}\text { 2. ED admission values of the EMS } \\
\text { group }\end{array}$ & $\begin{array}{l}\text { 3. ED admission values of the non-EMS } \\
\text { group }\end{array}$ \\
\hline SBP (mm Hg), mean (SD) & $139.0(32.1)$ & $140.6(27.0)$ & $139.6(29.7)$ \\
\hline Heart rate (bpm), mean (SD) & $89.2(22.5)$ & $84.5(19.0)[1]$ & $83.7(21.5)[2]$ \\
\hline Respiratory rate $(/ \mathrm{min}), \mathrm{n}=718$, mean $(\mathrm{SD})$ & $22.9(6.6)$ & $22.3(6.7)$ & $22.5(7.6)$ \\
\hline $\mathrm{SpO}_{2}(\%)$, mean $(\mathrm{SD})$ & $90.3(8.6)$ & $91.8(8.5)$ & $92.9(6.6)[3]$ \\
\hline $\mathrm{SpO}_{2}<90 \%, \mathrm{n}(\%)$ & $33(33.0)$ & $24(24.0)$ & $147(19.2)$ \\
\hline $\mathrm{SBP}<100 \mathrm{~mm} \mathrm{Hg}, \mathrm{n}(\%)$ & $6(6.5)$ & $6(6.0)$ & $39(5.1)$ \\
\hline SBP $100-119$ mm Hg, n (\%) & $17(18.3)$ & $17(17.0)$ & $145(18.9)$ \\
\hline SBP $120-160$ mm Hg, n (\%) & $46(49.5)$ & $53(53.0)$ & $432(56.4)$ \\
\hline $\mathrm{SBP}>160$ mm Hg, n (\%) & $24(25.8)$ & $24(24.0)$ & $150(19.6)$ \\
\hline
\end{tabular}

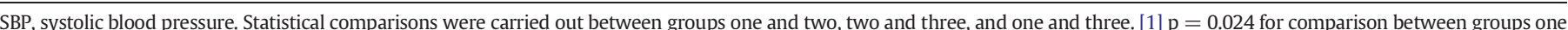

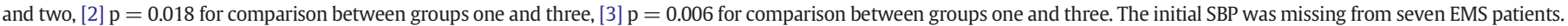

non-EMS patients. More than half of all the patients were normotensive or mildly hypertensive, while minority of patients was regarded as hypotensive. This finding is in line with previous data $[3,24]$. In addition no difference was observed in the most recently reported LVEF between EMS and non-EMS patients.

Despite the fact that EMS patients tended to have more comorbidities, no difference was seen in the in-hospital mortality between EMS and non-EMS patients. Neither did further analysis with propensity score change the result. The finding that EMS and non-EMS patients were almost equally stable on presentation to the ED and that no difference was seen in in-hospital mortality importantly demonstrates that all AHF patients should receive equally careful observation on presentation to the ED.

\section{Limitations}

This was a retrospective study. As we limited data to basic vital signs, we can assume that the results are reliable. Due to the fact that only 100 out of our total 873 AHF patients used EMS, our EMS cohort was relatively small. We also had missing data for some analysed parameters, especially the RR, which further restricted the number of values. Yet, it is a common finding that RR is the least systematically recorded vital sign $[25,26]$. The use of oxygen is not recorded in EMS and could not therefore be analysed.

The patients were initially relatively stable and therefore outcomes might have been different in more unstable patients. Patients passing ED e.g. directly to coronary care unit (CCU) were not included in the study. However, this did not exclude every acute coronary syndrome patient from our study population.

The data represents local policies and may not be universally generalizable. The pattern of prehospital medication administration may differ from that in other countries. Our EMS units are not equipped with intravenous furosemide, and only the most advanced EMS units, ALS and MICU, have the possibility for CPAP use and bronchodilators.

\section{Conclusion}

EMS use in AHF patients is predicted by clinical condition and earlier history of HF, but appears to be scarce. Few medications are administered in the prehospital setting, with nitrate spray and intravenous morphine being the most frequently administered ones. No differences are observed between non-EMS and EMS patients' vital signs on presentation to ED, LOS or in-hospital mortality. This underscores the need for equal attention to any AHF patient independent of the arrival mode. Moreover, as the clinical scenario of worsening CHF differs from cardiovascular emergencies like acute myocardial infarction or stroke with immediate onset, specific concepts of prehospital care have to be assessed in further studies.

\section{Funding}

The study was supported by the Department of Emergency Medicine and Services, Helsinki University Hospital and unrestricted research grants from Orion Pharma, Novartis and Servier.

\section{Conflict of interest}

The authors report no relationships that could be construed as a conflict of interest.

\section{References}

[1] J.A. Ezekowitz, M. Podder, A.F. Hernandez, et al., Arrival by ambulance in acute heart failure: insights into the mode of presentation from Acute Studies of Nesiritide in Decompensated Heart Failure (ASCEND-HF), BMJ Open 6 (2016), e010201-2015010201.

[2] Authors/Task Force Members, Document Reviewers, 2016 ESC Guidelines for the diagnosis and treatment of acute and chronic heart failure: the task force for the diagnosis and treatment of acute and chronic heart failure of the European Society of Cardiology (ESC). Developed with the special contribution of the Heart Failure Association (HFA) of the ESC, Eur. J. Heart Fail. (2016).

[3] A. Mebazaa, M.B. Yilmaz, P. Levy, et al., Recommendations on pre-hospital \& early hospital management of acute heart failure: a consensus paper from the Heart Failure Association of the European Society of Cardiology, the European Society of Emergency Medicine and the Society of Academic Emergency Medicine, Eur. J. Heart Fail. 17 (2015) 544-558.

[4] V.N. Mosesso Jr., J. Dunford, T. Blackwell, J.K. Griswell, Prehospital therapy for acute congestive heart failure: state of the art, Prehosp. Emerg. Care 7 (2003) 13-23.

[5] P. Plaisance, R. Pirracchio, C. Berton, E. Vicaut, D. Payen, A randomized study of outof-hospital continuous positive airway pressure for acute cardiogenic pulmonary oedema: physiological and clinical effects, Eur. Heart J. 28 (2007) 2895-2901.

[6] T. Kallio, M. Kuisma, A. Alaspaa, P.H. Rosenberg, The use of prehospital continuous positive airway pressure treatment in presumed acute severe pulmonary edema, Prehosp. Emerg Care 7 (2003) 209-213.

[7] P. Ponikowski, A.A. Voors, S.D. Anker, et al., 2016 ESC Guidelines for the diagnosis and treatment of acute and chronic heart failure: the task force for the diagnosis and treatment of acute and chronic heart failure of the European Society of Cardiology (ESC)Developed with the special contribution of the Heart Failure Association (HFA) of the ESC, Eur. Heart J. 18 (2016) 891-975.

[8] E.A. Stuart, Matching methods for causal inference: a review and a look forward, Stat Sci. 25 (2010) 1-21.

[9] V.P. Harjola, F. Follath, M.S. Nieminen, et al., Characteristics, outcomes, and predictors of mortality at 3 months and 1 year in patients hospitalized for acute heart failure, Eur. J. Heart Fail. 12 (2010) 239-248.

[10] A.P. Ambrosy, G.C. Fonarow, J. Butler, et al., The global health and economic burden of hospitalizations for heart failure: lessons learned from hospitalized heart failure registries, J. Am. Coll. Cardiol. 63 (2014) 1123-1133.

[11] C. Darling, J.S. Saczynski, D.D. McManus, D. Lessard, F.A. Spencer, R.J. Goldberg, Delayed hospital presentation in acute decompensated heart failure: clinical and patient reported factors, Heart Lung 42 (2013) 281-286.

[12] M. Takahashi, S. Kohsaka, H. Miyata, et al., Association between prehospital time interval and short-term outcome in acute heart failure patients, J. Card. Fail. 17 (2011) 742-747.

[13] J. Jaronik, P. Mikkelson, W. Fales, D.T. Overton, Evaluation of prehospital use of furosemide in patients with respiratory distress, Prehosp. Emerg. Care 10 (2006) 194-197.

[14] J.M. Kosowsky, M.D. Gasaway, S.L. Stephanides, M. Ottaway, M.R. Sayre, EMS transports for difficulty breathing: is there a potential role for CPAP in the prehospital setting? Acad. Emerg. Med. 7 (2000) 1165

[15] M. Eckstein, D. Suyehara, Ability of paramedics to treat patients with congestive heart failure via standing field treatment protocols, Am. J. Emerg. Med. 20 (2002) 23-25. 
[16] M.W. Hubble, M.E. Richards, R. Jarvis, T. Millikan, D. Young, Effectiveness of prehospital continuous positive airway pressure in the management of acute pulmonary edema, Prehosp. Emerg. Care 10 (2006) 430-439.

[17] K.A. Sporer, J.A. Tabas, R.K. Tam, et al., Do medications affect vital signs in the prehospital treatment of acute decompensated heart failure? Prehosp. Emerg. Care 10 (2006) 41-45.

[18] G. Bertini, C. Giglioli, A. Biggeri, et al., Intravenous nitrates in the prehospital management of acute pulmonary edema, Ann. Emerg Med 30 (1997) 493-499.

[19] S.P. Collins, A.B. Storrow, P.D. Levy, et al., Early management of patients with acute heart failure: state of the art and future directions-a consensus document from the SAEM/ HFSA acute heart failure working group, Acad. Emerg. Med. 22 (2015) 94-112.

[20] A.J. Singer, C. Emerman, D.M. Char, et al., Bronchodilator therapy in acute decompensated heart failure patients without a history of chronic obstructive pulmonary disease, Ann. Emerg. Med. 51 (2008) 25-34.

[21] C.A. Maak, J.A. Tabas, D.E. McClintock, Should acute treatment with inhaled beta agonists be withheld from patients with dyspnea who may have heart failure? J. Emerg. Med. 40 (2011) 135-145.
[22] W.F. Peacock, C.M. Cannon, A.J. Singer, B.C. Hiestand, Considerations for initial therapy in the treatment of acute heart failure, Crit. Care 19 (2015) (399-015-1114-3).

[23] M. Gardtman, L. Waagstein, T. Karlsson, J. Herlitz, Has an intensified treatment in the ambulance of patients with acute severe left heart failure improved the outcome? Eur. J. Emerg. Med. 7 (2000) 15-24.

[24] M. Gheorghiade, W.T. Abraham, N.M. Albert, et al., Systolic blood pressure at admission, clinical characteristics, and outcomes in patients hospitalized with acute heart failure, JAMA 296 (2006) 2217-2226.

[25] J. Nurmi, V.P. Harjola, J. Nolan, M. Castren, Observations and warning signs prior to cardiac arrest. Should a medical emergency team intervene earlier? Acta Anaesthesiol. Scand. 49 (2005) 702-706.

[26] J. Tirkkonen, J. Yla-Mattila, K.T. Olkkola, H. Huhtala, J. Tenhunen, S. Hoppu, Factors associated with delayed activation of medical emergency team and excess mortality: an Utstein-style analysis, Resuscitation 84 (2013) 173-178. 